\title{
The tension-compression asymmetry of martensite phase transformation in a metastable $\mathrm{Fe}_{40} \mathrm{Co}_{20} \mathrm{Cr}_{20} \mathrm{Mn}_{10} \mathrm{Ni}_{10}$ high-entropy alloy
}

\author{
Xinglong $\mathrm{An}^{1}$, Zhangwei Wang ${ }^{2^{*}}$, Song $\mathrm{Ni}^{1}$ and Min Song ${ }^{1^{*}}$
}

\begin{abstract}
The microstructural evolution of a metastable face centered cubic (FCC) $\mathrm{Fe}_{40} \mathrm{Co}_{20} \mathrm{Cr}_{20} \mathrm{Mn}_{10} \mathrm{Ni}_{10}$ high-entropy alloy (HEA) under both tension and compression is systemically investigated. The results show much higher level of martensite phase transformation from FCC structure to hexagonal closed packed (HCP) structure under compression than tension, indicating a distinct tension-compression asymmetry. The compressive tests underwent higher true stresses, which further provided stronger driving forces to trigger the phase transformation than those in tensile tests. Except for the martensite phase transformation, dislocation planar slip prevails in both tension and compression, along with the occasional formation of mechanical twins. Dislocation slip dominates the whole tensile deformation, while both dislocation motions and martensite phase transformation play critical roles in the compressive deformation. The martensite phase transformation is preferred to nucleate at grain or subgrain boundaries due to a medium stacking fault energy (SFE) of $\sim 20 \mathrm{~mJ} \mathrm{~m}^{-2}$. The formation of HCP phase via partial dislocation emission from low-angle grain boundaries offers additional pathways for martensite phase transformation. Our study thus remarkably benefits the understanding of the deformation mechanisms of metastable HEAs.
\end{abstract}

Keywords: high-entropy alloy, tension-compression, martensite phase transformation, stacking fault energy

\section{INTRODUCTION}

High-entropy alloy (HEA), as a new concept for alloy design, has gained tremendous attention due to its intrinsic compositional variability and attractive properties [1-7]. It is well established that tuning chemical compositions to vary the stacking fault energy (SFE) in HEAs is an efficient way to enhance the mechanical properties, since the SFE is a critical intrinsic factor to determine the deformation mechanisms in face centered cubic (FCC) HEAs [8,9]. The desirable deformation mechanisms in FCC HEAs include microband-induced plasticity (MBIP) [10-12], twining-induced plasticity (TWIP) [13-15], and transformation-induced plasticity (TRIP) [9,16-18], all of which are able to overcome strength-ductility trade-off barrier. In this regard, the strategies of designing metastable FCC HEAs have shown significant potential to achieve high-performance HEAs. This is because the strain hardening of metastable HEAs is dominated by the TRIP effect, with joint activation of the TWIP effect depending on SFEs [19]. Normally, for HEAs, the TRIP effect is expected to occur when the SFE is below $20 \mathrm{~mJ} \mathrm{~m}^{-2}$, while the TWIP effect is typically observed when the SFE is in the range of $18-35 \mathrm{~mJ} \mathrm{~m}^{-2}$ [8].

One of the key issues for metastable HEAs is to clarify the underlying deformation and strain hardening mechanisms. The martensite phase transformation from FCC structure to hexagonal closed packed (HCP) structure occurs in metastable HEAs upon deformation, which is achieved via the dissociation of dislocations [20]. Wang et al. [21] reported that the formation of HCP plates involves cross-slip of stacking faults (SFs) and mutual interactions among dislocations in a $\mathrm{Fe}_{49.5} \mathrm{Mn}_{30} \mathrm{Co}_{10} \mathrm{Cr}_{10} \mathrm{C}_{0.5}$ (at.\%) HEA. Chen et al. [22] further found that HCP phase formed at the stacking-fault network boundaries in the FCC phase, causing strong strain hardening in a $\mathrm{Cr}_{20} \mathrm{Mn}_{6} \mathrm{Fe}_{34} \mathrm{Co}_{34} \mathrm{Ni}_{6}$ (at.\%) HEA. In addition, cryogenic loading conditions, such as 77 and $4.2 \mathrm{~K}$, can significantly promote the martensite phase transformation due to the low SFE and high flow stress $[18,21]$. Previous studies focused on the microstructural evolution and deformation mechanism under tensile test of metastable HEAs;

\footnotetext{
${ }^{1}$ State Key Laboratory of Powder Metallurgy, Central South University, Changsha 410083, China

${ }^{2}$ Max-Planck-Institut für Eisenforschung, Max-Planck-Str. 1, Düsseldorf 40237, Germany

*Corresponding authors (emails: zh.wang@mpie.de (Wang Z); msong@csu.edu.cn (Song M))
} 
however, deformation behavior under compression has not been reported in detail. Whether the compression would induce different deformation behaviors of metastable HEAs, such as the variation of twinning and phase transformation activity, remains unknown, though it is critical to understand the strain hardening of HEAs.

In the present work, we report mechanical responses of a single FCC metastable $\mathrm{Fe}_{40} \mathrm{Co}_{20} \mathrm{Cr}_{20} \mathrm{Mn}_{10} \mathrm{Ni}_{10}$ (at.\%) HEA with a medium SFE of $20 \mathrm{~mJ} \mathrm{~m}^{-2}$ under both tension and compression. A severe tension-compression asymmetry was detected, of which much higher levels of martensite phase transformation and larger true stresses were exhibited in compressive tests than those in tensile tests. The microstructural evolutions under both tests at various strain levels were systematically characterized via electron backscatter diffraction (EBSD), electron channeling constant imaging (ECCI), and transmission electron microscopy (TEM). The underlying mechanisms for the tension-compression asymmetry were uncovered, in terms of the formation pathways of martensite and twining at atomic scales. Our study provides valuable insights into the understanding of mechanical behaviors of metastable HEAs, and thus, yields a strong benefit to the design of HEAs with outstanding properties.

\section{EXPERIMENTAL SECTION}

A non-equiatomic HEA with a nominal composition of $\mathrm{Fe}_{40} \mathrm{Co}_{20} \mathrm{Cr}_{20} \mathrm{Mn}_{10} \mathrm{Ni}_{10}$ (at.\%) was prepared via a vacuum induction melting technique with high purity raw metals $(>99.5 \%)$. The as-cast alloy was cold rolled to a thickness reduction of $80 \%$, followed by annealing at $1100^{\circ} \mathrm{C}$ for $1 \mathrm{~h}$. Tensile and compressive tests were performed on an Instron 3369 testing system with a strain rate of $10^{-3} \mathrm{~s}^{-1}$ at room temperature. Deformation microstructures at various true strains $(0.1,0.4$, and 0.6$)$ under both tension and compression were examined by scanning electron microscopy (SEM) and TEM. T1, T2 and T3, and C1, C2 and $\mathrm{C} 3$ represented the samples after tension or compression to low (0.1), medium (0.4), and high (0.6) strains, respectively. EBSD and electron channeling constant (ECC) imaging were conducted in Zeiss sigma 500 SEM and Zeiss Merlin SEM, respectively. The deformation substructures were further investigated via Titan G2 Cs-corrected TEM operated at $300 \mathrm{kV}$. Samples for SEM were prepared by mechanical polishing, as seen in Ref. [23]. The TEM thin foils cut from the deformed samples were prepared by mechanical grounding and then electropolishing in a twin-jet machine consisting of 10 vol.\% perchloric acid and 90 vol.\% methanol at the voltage of $35 \mathrm{~V}$ and a temperature of $-45^{\circ} \mathrm{C}$. SFE was calculated through the widths of Shockley partial dislocations in a $1 \%$ pre-strained HEA, which were measured from the dark field image with the beam direction near [111] zone axis using $g=<220>$ vector [24]. The average width was calculated based on five measurements. The shear modulus was measured from nano-indentation, while the lattice parameters of HEA were determined by X-ray diffraction (XRD) calculated through Jade software.

\section{RESULTS}

\section{The tension-compression asymmetry}

The initial microstructures of the $\mathrm{Fe}_{40} \mathrm{Co}_{20} \mathrm{Cr}_{20} \mathrm{Mn}_{10} \mathrm{Ni}_{10}$ HEA are shown in Fig. 1. The HEA exhibits a single FCC structure based on EBSD phase map and XRD pattern, with an average grain size of $\sim 40 \mu \mathrm{m}$ (see Fig. $1 \mathrm{a}$ and $\mathrm{b}$ ). ECC image in Fig. 1c shows the existence of annealing twins in a fully recrystallized grain. The single FCC structure is further confirmed by a high magnification ECC image (Fig. 1d), in which only very low density of dislocations and SFs exist in this annealed HEA.

Fig. 2 shows a dark field TEM image of a dissociated dislocation in a $1 \%$ pre-strained HEA. The separation distance $(d)$ of this partial dislocation and the angle $(\theta)$ between the Burgers vector and the dislocation line were measured from the image. Thus, the SFE can be calcu-

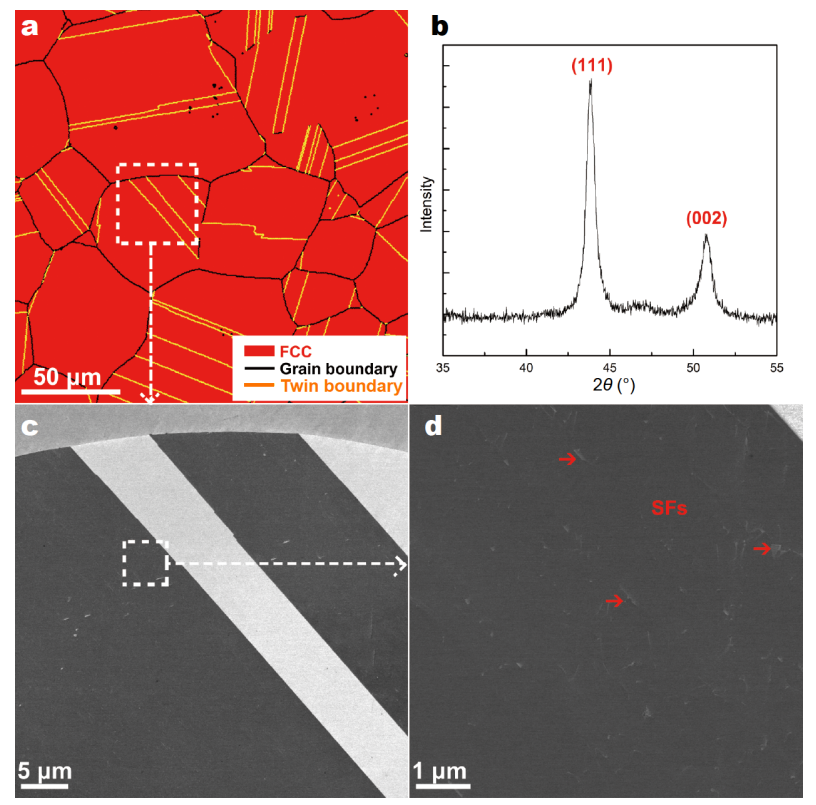

Figure 1 Microstructures of the HEA in the homogenized state. Both EBSD phase map (a) and XRD (b) confirm the single FCC structure in this alloy. (c) Low-magnification ECC image corresponding to the identical region marked in (a); and (d) high-magnification ECC image corresponding to the identical region marked in (c). 


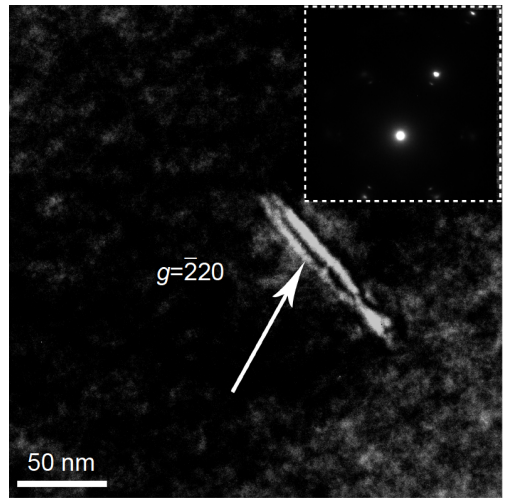

Figure 2 A dark field TEM image showing a dissociated dislocation in the $1 \%$ pre-strained HEA under the two-beam condition.

lated through the following equation [24]:

$\gamma=\frac{G b_{\mathrm{p}}^{2}}{8 \pi d} \cdot \frac{2-v}{1-v}\left(1-\frac{2 v \cos 2 \theta}{2-v}\right)$,

where $G=83 \mathrm{GPa}$ is the shear modulus, $v=0.33$ is Poisson's ratio, $b_{\mathrm{p}}$ is the Burgers vector of partial dislocation. $b_{\mathrm{p}}$ is obtained by $b_{\mathrm{p}}=\frac{1}{6}<112>a$, where $a$ is the lattice parameter $(a=0.359 \mathrm{~nm})$. The experimental result shows that the SFE of this HEA is $20 \pm 4 \mathrm{~mJ} \mathrm{~m}^{-2}$, which is in the TRIP-TWIP HEA region [8].

Fig. 3 shows the engineering stress-strain curves, true stress-strain curves, and strain hardening rate curves of the HEA under tensile and compressive tests. Both tensile and compressive curves exhibit nearly the same yield point, which gives the value of $\sim 250 \mathrm{MPa}$. On the contrary, the ultimate engineering compressive stress of the HEA can reach over $3000 \mathrm{MPa}$, which is much larger than the ultimate tensile stress (673 MPa), suggesting a distinct tension-compression asymmetry. The strain hardening rate curves (inset in Fig. 3b) show the similar asymmetry, in which the strain hardening rates are higher in the compressive curve than those in the tensile curve over the whole deformation regime. Similar tensioncompression asymmetry was also observed previously in NiTi shape memory alloys $[25,26]$. To further understand the difference between the tensile and compressive behaviors, three different deformation stages (with true strains of $0.1,0.4$ and 0.6 ) were chosen to characterize the microstructures. The engineering and true stresses at these different stages are given in Table 1.

The corresponding deformation microstructures at different strains via tension and compression are revealed by EBSD phase maps overlapped with image quality (IQ) maps in Fig. 4. Deformation-induced martensitic phase transformation from FCC to HCP was detected in both tests, yet the content of the martensitic phase transformation varies. During the tensile test, no phase transformation is found at the low and medium strains (T1 and T2 samples), while $~ 9 \%$ HCP phase is detected until the strain goes up to 0.6 (T3 sample), as seen in Fig. 4a-c. At the early stage of compressive deformation, no HCP phase can be detected in the $\mathrm{C} 1$ sample either (Fig. $4 \mathrm{~d}$ ). With the increase of strain to $0.4, \sim 9 \%$ HCP phase exists in the $\mathrm{C} 2$ sample (Fig. 4e), and the fraction of HCP phase reaches up to $41 \%$ in the $\mathrm{C} 3$ sample when the strain raises to 0.6 (Fig. 4f). Overall, the HEA undergoes far stronger martensitic phase transformation in compression when compared with tension, which is in good agreement with the higher true stress in compression than tension, as shown in Fig. 3 and Table 1.

\section{Deformation substructure evolution under tension}

Fig. 5 shows the ECC image and bright field (BF) TEM image of the T1 sample (true strain of 0.1). Linear arrays consisting of numerous dislocations are seen in Fig. 5a, suggesting a typical planar slip. The high-magnification TEM image depicts the existence of single dislocations.
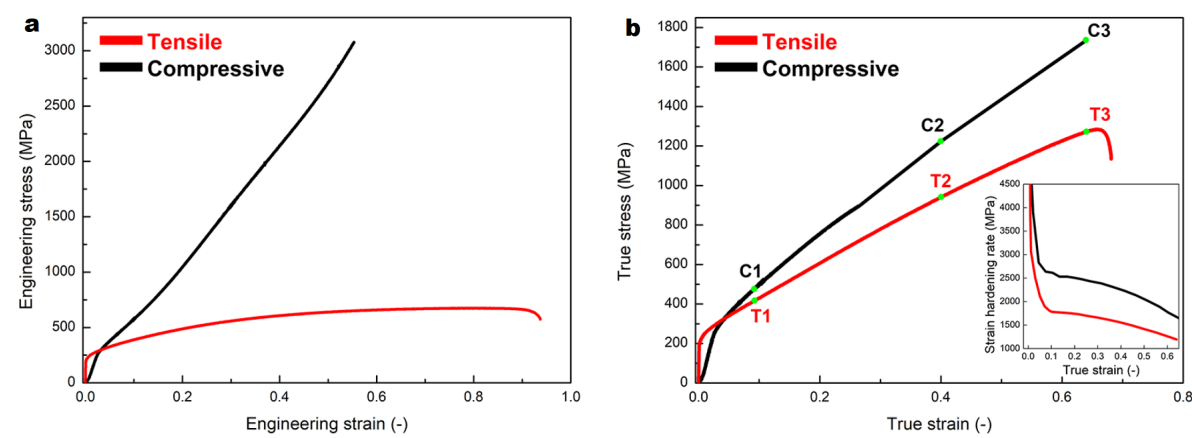

Figure 3 The engineering (a) and true stress-strain (b) curves and corresponding strain hardening rate curves of the HEA under both tension and compression. 
Table 1 The engineering and true stresses in HEA at low (0.1), medium (0.4) and high (0.6) strains during tension and compression

\begin{tabular}{ccccc}
\hline & True strain & $\begin{array}{c}\text { Engineering } \\
\text { strain }\end{array}$ & $\begin{array}{c}\text { True stress } \\
(\mathrm{MPa})\end{array}$ & $\begin{array}{c}\text { Engineering } \\
\text { stress (MPa) }\end{array}$ \\
\hline $\mathrm{T} 1$ & \multirow{2}{*}{0.1} & 0.1 & 421 & 384 \\
$\mathrm{C} 1$ & & 0.09 & 483 & 534 \\
$\mathrm{~T} 2$ & & 0.49 & 941 & 630 \\
$\mathrm{C} 2$ & 0.4 & 0.33 & 1223 & 1764 \\
$\mathrm{~T} 3$ & & 0.90 & 1273 & 670 \\
$\mathrm{C} 3$ & 0.6 & 0.47 & 1735 & 2527 \\
\hline
\end{tabular}

Based on the corresponding selective area diffraction (SAED) pattern, dislocation lines are parallel to two different $\{111\}$ planes with an angle of $70.5^{\circ}$. SFs can be observed besides of planar slip, which were formed via the dissociation of dislocations [27] (see the arrowed ones in Fig. 5b). Dislocation slip dominated at this deformation stage.

Fig. 6 shows the microstructure of the T2 sample based on ECCI and TEM observations. Compared with the T1 sample, the martensite phases with lamellar shape were formed, as pointed by the green arrows in Fig. 6a. The high-resolution TEM (HRTEM) image of Fig. 6a shows the microstructure of the HCP lamellar at the atomic scale. The orientation relationships between FCC matrix and HCP lamellae obey the Shoji-Nishiyama (S-N) rule [28], which can be described as $(0001)_{\mathrm{HCP}} / /\{111\}_{\mathrm{FCC}}$ and $\left.\langle 11 \overline{2} 0\rangle_{\mathrm{HCP}} / /<110\right\rangle_{\mathrm{FCC}}$. The insert of Fig. $6 \mathrm{~b}$ shows the atomic structure of the phase boundary by the fast Fourier transform (FFT)-filtered HRTEM image. The burgers circuit shows a $30^{\circ}$ Shockley partial dislocation with Burgers vector of $\frac{1}{6}[11 \overline{2}]$ at the phase boundary. The martensite transformation from FCC to HCP can occur through Shockley partial dislocation gliding on every other $\{111\}$ planes, which eventually, transform the atom stacking sequence from ...ABCABC... (FCC) to ...ABABAB... (HCP) [20,28]. The SF marked via yellow triangle was also observed. The formation of HCP phases is usually considered as regularly overlapped SFs [28]. At this deformation stage, dislocation slip still dominated, while martensite transformation was triggered.

Deformation substructures after a very high tensile strain of 0.6 (T3 sample) are shown in Fig. 7. With the increase of strain, more lamellae can be observed in Fig. $7 \mathrm{a}$, together with the existence of very high density of dislocations. In addition, shear bands are also observed in Fig. $7 \mathrm{a}$, and these shear bands are connected with plenty of HCP lamellae, as revealed by the magnified inset image

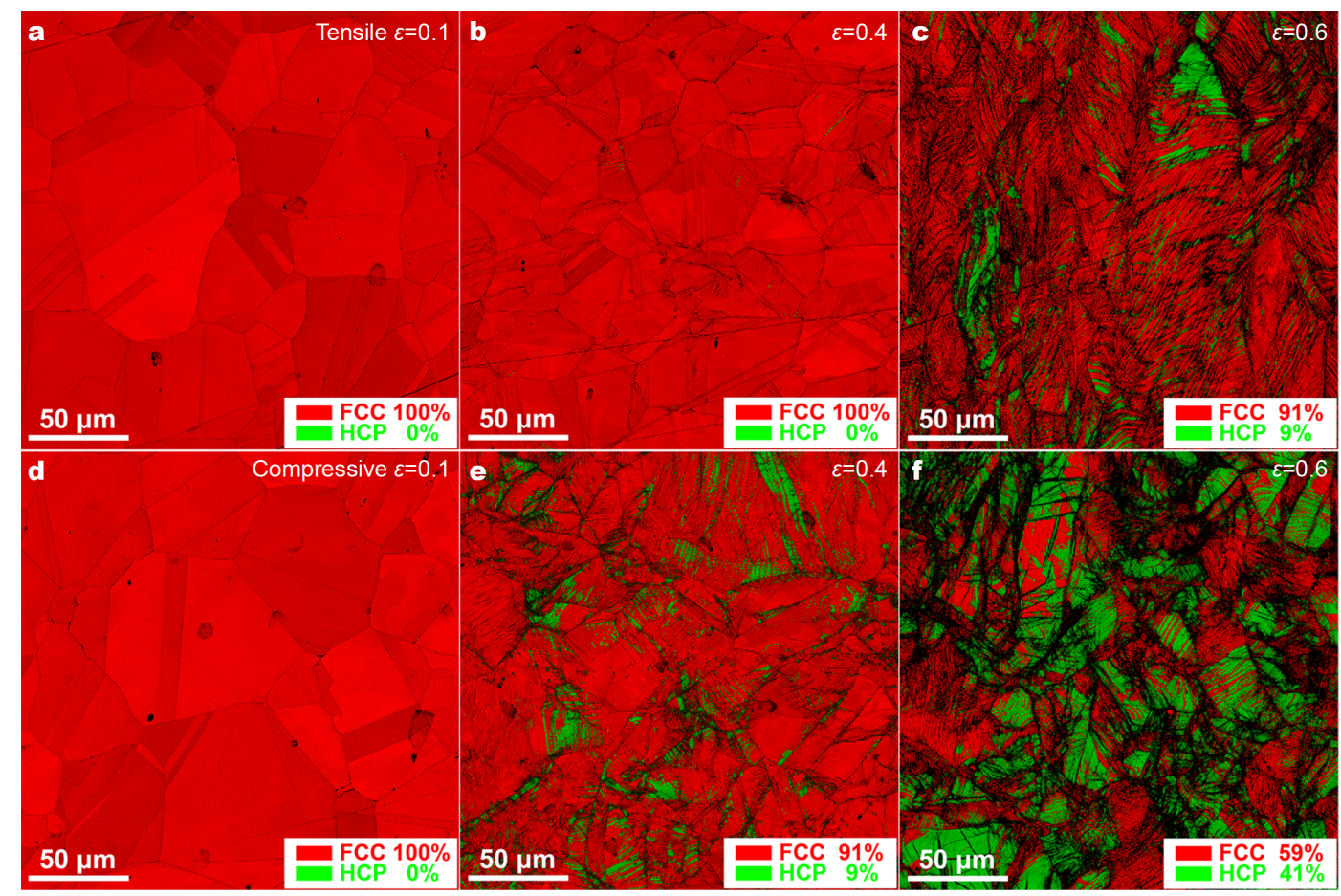

Figure 4 EBSD phase maps of $\mathrm{Fe}_{40} \mathrm{Co}_{20} \mathrm{Cr}_{20} \mathrm{Mn}_{10} \mathrm{Ni}_{10}$ HEA at various strains of $0.1,0.4$ and 0.6 via $(\mathrm{a}-\mathrm{c}$ ) tension and (d-f) compression. (a-c) show EBSD maps of T1, T2, and T3, respectively, and (d-f) show EBSD maps of $\mathrm{C} 1, \mathrm{C} 2$, and $\mathrm{C} 3$, respectively. 


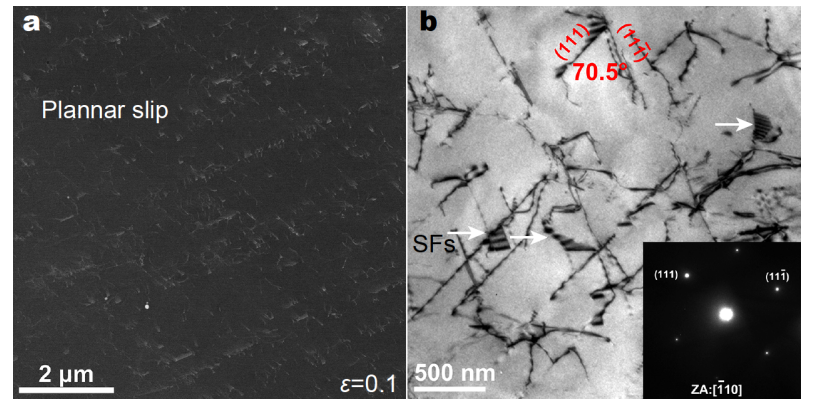

Figure 5 The ECC image (a) and BF TEM image (b) with its corresponding SAED in the right bottom corner showing the dislocation substructures of the T1 sample (tensile strain of 0.1 ).

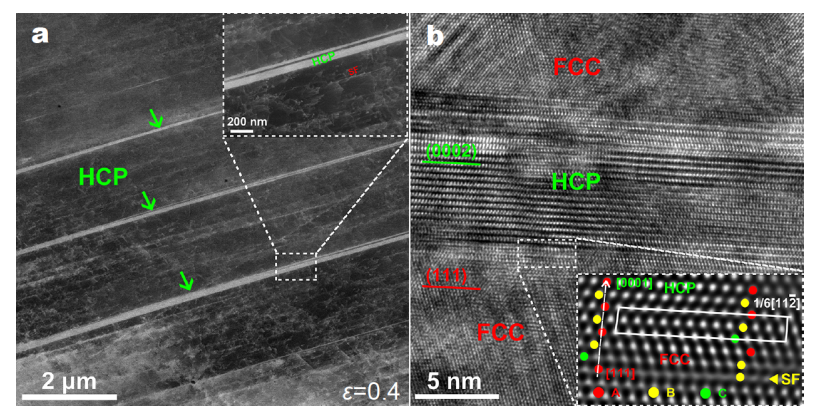

Figure 6 The ECC image (a) and HRTEM image (b) showing the deformation substructures of the T2 sample (tensile strain of 0.4 ). Green arrows indicate the martensite lamellae and the FFT-filtered image shows the phase boundary, as inserted in (b).

in Fig. 7a. The BF TEM image in Fig. $7 \mathrm{~b}$ confirms the connection between shear bands and HCP lamellae, as pointed by the green arrows. Also, the TEM image indicats that dislocation cells were formed inside the shear bands due to dislocation tangles. It is worth noting that the existence of high density of dislocations at the shear band boundaries was resulted from their abilities to trap dislocations. These boundaries acted as dislocation resources, which emitted partial dislocations, and thus, triggered the martensite phase transformation. As a result, HCP lamellae formed to connect with the shear bands (see Fig. 7a and b).

Several thin lamellar plates were detected by the BF TEM image in Fig. 7c (arrowed), and the corresponding SAED pattern indicates that these lamellae are mechanical twins. In contrast to the strong martensite phase transformation, deformation-induced twinning was rarely observed even at this high level of strain. Fig. $7 d$ shows that the HCP lamellae can be further formed on both sides of the grain boundaries, as marked via yellow dotted line. These HCP lamellae are all connected along the grain boundary. The SAED pattern shows a $13.9^{\circ}$

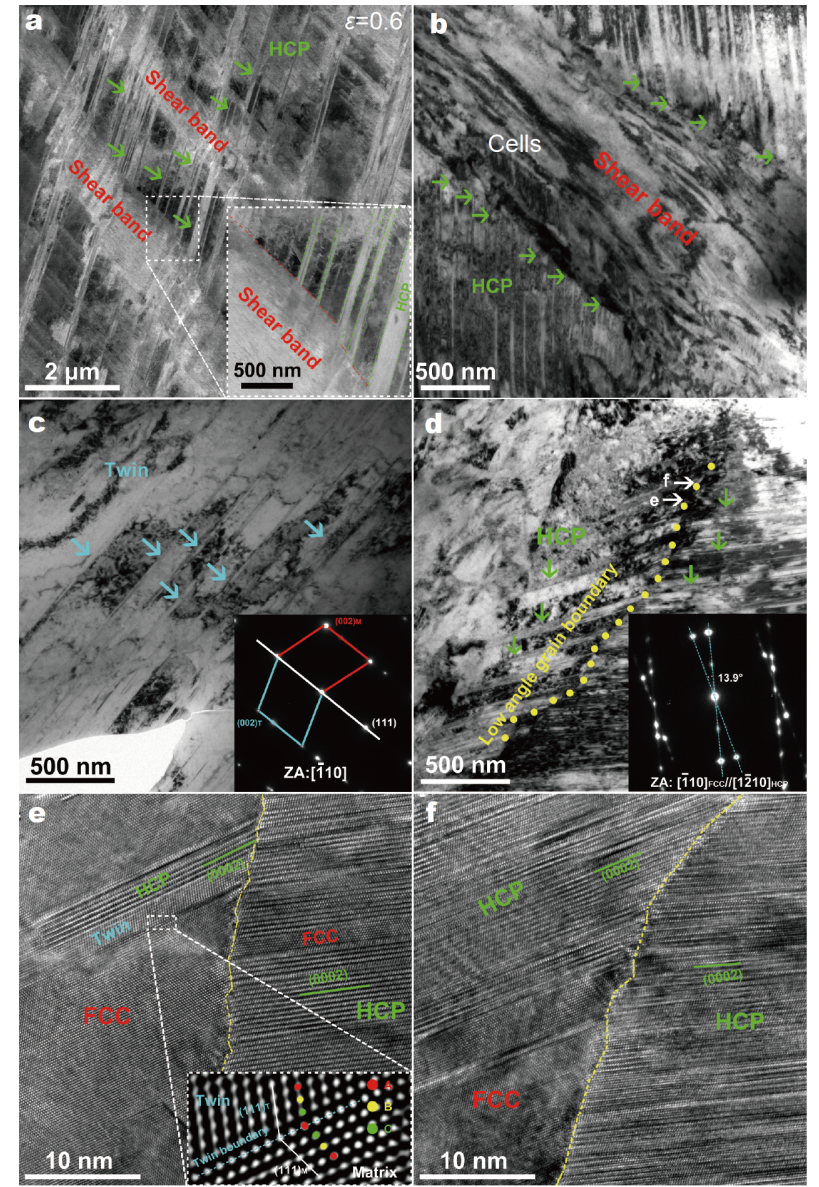

Figure 7 The ECC images and TEM images showing the deformation microstructures of the T3 sample (tensile strain of 0.6). Both ECC image (a) and TEM image (b) show the martensite HCP lamellae (marked via green arrows) connected with shear bands. (c) TEM image with its corresponding SAED pattern shows the deformation twinning (marked via blue arrows). (d) TEM image with its corresponding SAED pattern shows several HCP plates (marked via green arrows) emitted from a low-angle grain boundary (marked via yellow dotted line). (e, f) HRTEM images showing the substructures at the low-angle grain boundary in (d) at atomic scales.

misorientation between the two sides of this boundary viewed from the [110] zone axis, suggesting a low-angle grain boundary here.

Two HRTEM images of this boundary are shown in Fig. $7 \mathrm{e}$ and $\mathrm{f}$. In Fig. $7 \mathrm{e}$, the right side region of the boundary is almost all HCP structure, while the left side region is FCC structure. Two sides are completely separated by the boundary. Another interesting observation is that an FCC twin was observed in the left side region. The inserted FFT-filtered image shows the atom stacking sequence of the matrix and twin through the [111] direction. The twin boundary was marked via blue dashed line. This twin was connected with FCC matrix at the down 
side and HCP lamellae at the upper side. Both twin and HCP lamellae were formed via partial dislocations emitted from the low-angle grain boundary. Fig. $7 f$ shows another area of this boundary. HCP blocks exist on both right and left sides, since the partial dislocations can be emitted to both sides to form the HCP lamellae. In short, the dislocation slip dominates the whole tensile deformation regime, while the deformation-induced HCP takes place at the medium and later stages of deformation, along with the occasional occurrence of mechanical twinning.

\section{Deformation substructure evolution under compression}

Fig. 8 shows the microstructure of compressive $\mathrm{C} 1$ (Fig. 8a-c), C2 (Fig. 8d-f), and C3 samples (Fig. 8g-i) corresponding to true strains of $0.1,0.4$, and 0.6 , respectively. In the $\mathrm{C} 1$ sample, except for the planar slip, more SFs formed than that in the T1 sample with the same strain, when comparing Fig. 8a with Fig. 5a. The inset in Fig. 8a with high magnification indicates that these SFs connect with each other and form plate-like structures, which are the initial stage of the martensite transformation. Fig. $8 \mathrm{~b}$ reveals the interaction between

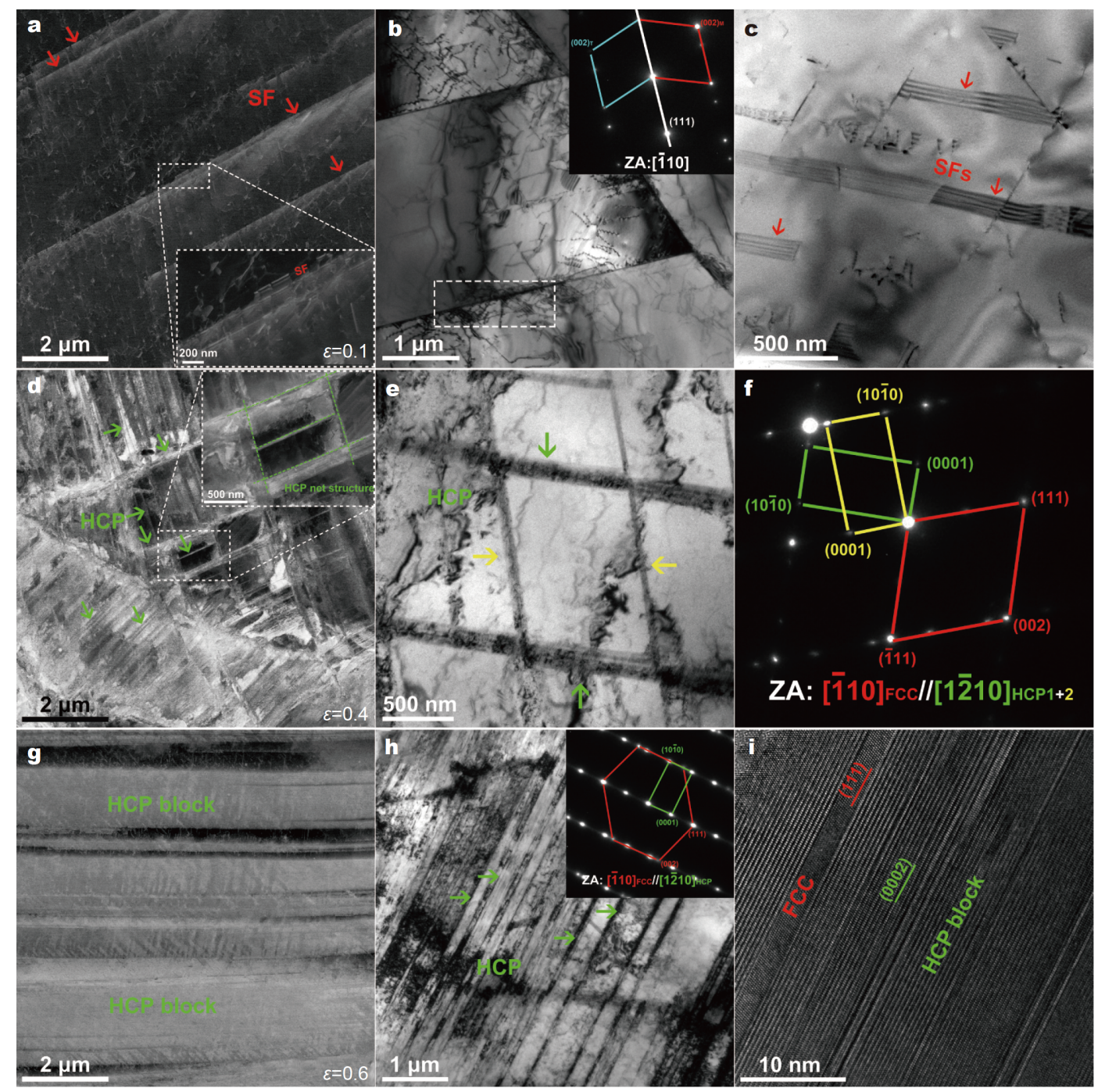

Figure 8 The ECC and TEM images of HEA at compressive strains of (a-c) 0.1 (C1 sample), (d-f) 0.4 (C2 sample) and (g-i) 0.6 (C3 sample). (a) ECC images and (b, c) BF TEM images showing SFs and planar dislocations at a strain of 0.1 . SFs are marked by red arrows and the inset in (a) shows the detailed morphology of SFs. The white rectangular box marked in (b) showing dislocations tangled at an annealing twin boundary. (d) ECC images, (e) BF TEM image, and (f) corresponding SAED pattern showing HCP net structures at a strain of 0.4. Two directional HCP lamellae with S-N relationship are marked via green and yellow arrows in (e) and (f). (g) ECC image, (h) BF TEM image with its corresponding SAED pattern, and (i) HRTEM image showing coarse HCP blocks at a strain of 0.6. The inserted SAED pattern in (h) indicates the dual phase of FCC and HCP structures. Green arrows indicate the coarse HCP block. 
dislocations and annealing twin boundaries by TEM analysis. An annealing twin is indicated by the SAED pattern (see the inset in Fig. 8b). The white dash box in Fig. $8 \mathrm{~b}$ marks the pile-ups of tangled dislocations at this twin boundary. The BF TEM image in Fig. 8c clearly records the dissociation of full dislocations to partial dislocations, to form SFs. At this deformation stage, dislocation slip also dominated in the $\mathrm{C} 1$ sample, whereas more SFs were seen when comparing with the T1 sample under the same strain.

Fig. $8 \mathrm{~d}-\mathrm{f}$ show the microstructure of $\mathrm{C} 2$ samples. $\mathrm{Nu}-$ merous HCP lamellae are seen in the ECC image (Fig. 8d), which is consistent with the EBSD observation (Fig. 4e). As shown in the inset in Fig. 8d, two directional HCP phases interact with each other, building HCP net structures. Such net structures of HCP plates are further revealed by the high-magnified TEM image in Fig. 8e. The angle between two directional HCP lamellae marked via green and yellow arrows is around $70.5^{\circ}$, which is exactly the angle between two different $\{111\}$ planes. The SAED pattern from the junction area between two HCP lamellae is shown in Fig. 8f. Both two HCP lamellae have the same S-N relationship with the FCC matrixes yet on different $\{111\}$ planes, which arises from the activation of two directional partial slip systems. At the true strain of 0.4 , stronger martensite phase transformation was observed in the compressive sample than that in the tensile one.

Fig. 8g-i show the microstructure of the $\mathrm{C} 3$ sample. Coarse HCP blocks with a thickness of several micrometers are observed in Fig. 8g. The BF TEM in Fig. $8 \mathrm{~h}$ shows that plenty of lamellae exist in the matrix, which consist of both FCC and HCP phases, as proved by the SAED pattern on the right upper corner of Fig. $8 \mathrm{~h}$. The HRTEM in Fig. 8i confirms that the thin plates are FCC phase, which exists in the HCP matrix. This suggests that FCC structure is the untransformed one inside the transformed HCP structure, thus exhibiting as the very thin plate structures with the thickness less than $10 \mathrm{~nm}$. In summary, the impressive martensite phase transformation takes place during the compression, producing up to $41 \%$ HCP phase after the tests, which indicates that phase transformation plays a key role in the compressive deformation.

\section{DISCUSSION}

\section{Overview of the microstructural evolution}

Our study again proves the excellent mechanical performance of metastable HEAs, of which the true tensile strain is close to 0.7 with a very high tensile true stress of $1300 \mathrm{MPa}$. Such high strength and large ductility are ascribed to the activation of multiple strain hardening mechanisms, as shown in Figs 5-8. Fig. 9 summarizes the deformation microstructure evolution of the HEA with the increase of strain via schematic illustration and TEM images. The HEA has a single FCC structure in the undeformed state with the lack of HCP phase. Upon deformation, the planar dislocations dominated the early deformation stage, while the martensite phase transformation occurred later. Besides, multiple widely extended SFs and deformation twinning also took place to coordinate the local strain during deformation. In a more micro level, the planar dislocations generated and slipped at the early deformation stage. With further deformation, these dislocations were tangles and dissociated into partial dislocations to form SFs. The partial dislocations regularly glided on every other $\{111\}$ planes and eventually changed the atom sequences to form HCP lamellae. These lamellae combined with each other to form the HCP block.

Dislocation slip, as the most common deformation mechanism, is usually stored and arranged in ordered patterns during deformation. The motions of dislocations are patterned with two modes: planar slip and wave slip [29]. The wave slip is caused by frequent cross-slip, while the planar slip is promoted by low SFE, or high internal friction stress via short range ordering or solute content $[10,30]$. In our HEA, the planar slip along $\{111\}$ planes are seen in Figs $5 \mathrm{a}$ and $8 \mathrm{a}$ under both tension and compression. The HEAs with five major elements are known for the very high lattice friction stress due to the mass solid solution strengthening $[4,30]$. In addition, the current HEA has a relatively low SFE of $\sim 20 \mathrm{~mJ} \mathrm{~m}^{-2}$, as shown in Fig. 2. The observation of many SFs and the followed martensite phase transformation are also the evidence of low SFE. Thus, planar slip is facilitated by the low SFE and the large internal friction stress in this HEA.

Deformation twinning has been widely reported in FCC materials with SFE in the range of $18-35 \mathrm{~mJ} \mathrm{~m}^{-2}$ [31]. The SFE of our HEA, as noted earlier, is $\sim 20 \mathrm{~mJ} \mathrm{~m}^{-2}$, very close to the critical point, which explains that only rare mechanical twins can be seen. Though very rare, two different types of twinning are detected (Fig. $7 c$ and e). The first type of twinning is formed in a coarse grain (Fig. 7c), while the second one is formed in a nano-size band structure (Fig. 7e). Once a dislocation source is obtained in the grain interior of coarse grain, twins can be formed via partial dislocations with the same Burgers vector gliding on every $\{111\}$ plane [32]. The nano twins 
in Fig. 7e were formed via partial dislocations emitted from grain boundary, a different mechanism from previous one [33]. The deformation induced the formation of low-angle boundaries, which contained a high density of dislocations. These subgrain boundaries can be considered as the dislocation resource to emit partial dislocations [34], which triggers the formation of twins in Fig. 7 e.

The FCC to HCP martensite phase transformation occurs in FCC materials when the SFE is below $20 \mathrm{~mJ} \mathrm{~m}^{-2}$ [8]. Upon deformation, partial dislocations can be either dissociated from $60^{\circ}$ full dislocations or screw dislocations [35]. The FCC to HCP martensite phase transformation can occur through partial dislocations gliding on ever other $\{111\}$ plane, as seen in Fig. 6 [28]. In addition to this previously reported mechanism, a different formation pathway of HCP phases is detected (Fig. 7a and e). Partial dislocations can be emitted from low-angle grain boundaries, which act as nucleation sites for HCP phase. In this case, whether to form HCP or FCC twin is completely determined by partial dislocation behavior. If the partial dislocations emitted from grain boundary are gliding on every $\{111\}$ plane, the FCC twin will be formed. Otherwise, if the partial dislocations are gliding on every other $\{111\}$ plane, the HCP lamellae will thus be formed.

\section{The origin of tension-compression asymmetry}

By comparing the tension and compression behaviors, the fundamental difference concentrates on the martensite phase transformation, while other deformation substructures, such as dislocation slip and mechanical twinning, remain very similar, as illustrated in Fig. 9. EBSD phase maps in Fig. 4 show that a far higher extent of phase transformation is seen in compression than tension, which corresponds to a much higher true stress and strain hardening rate (see Table 1 and Fig. 3). For example, at a true strain of 0.6 , HCP phase fraction is $9 \%$ under tension and $41 \%$ under compression, which corresponds to the true stress of 1200 and $1700 \mathrm{MPa}$, re-
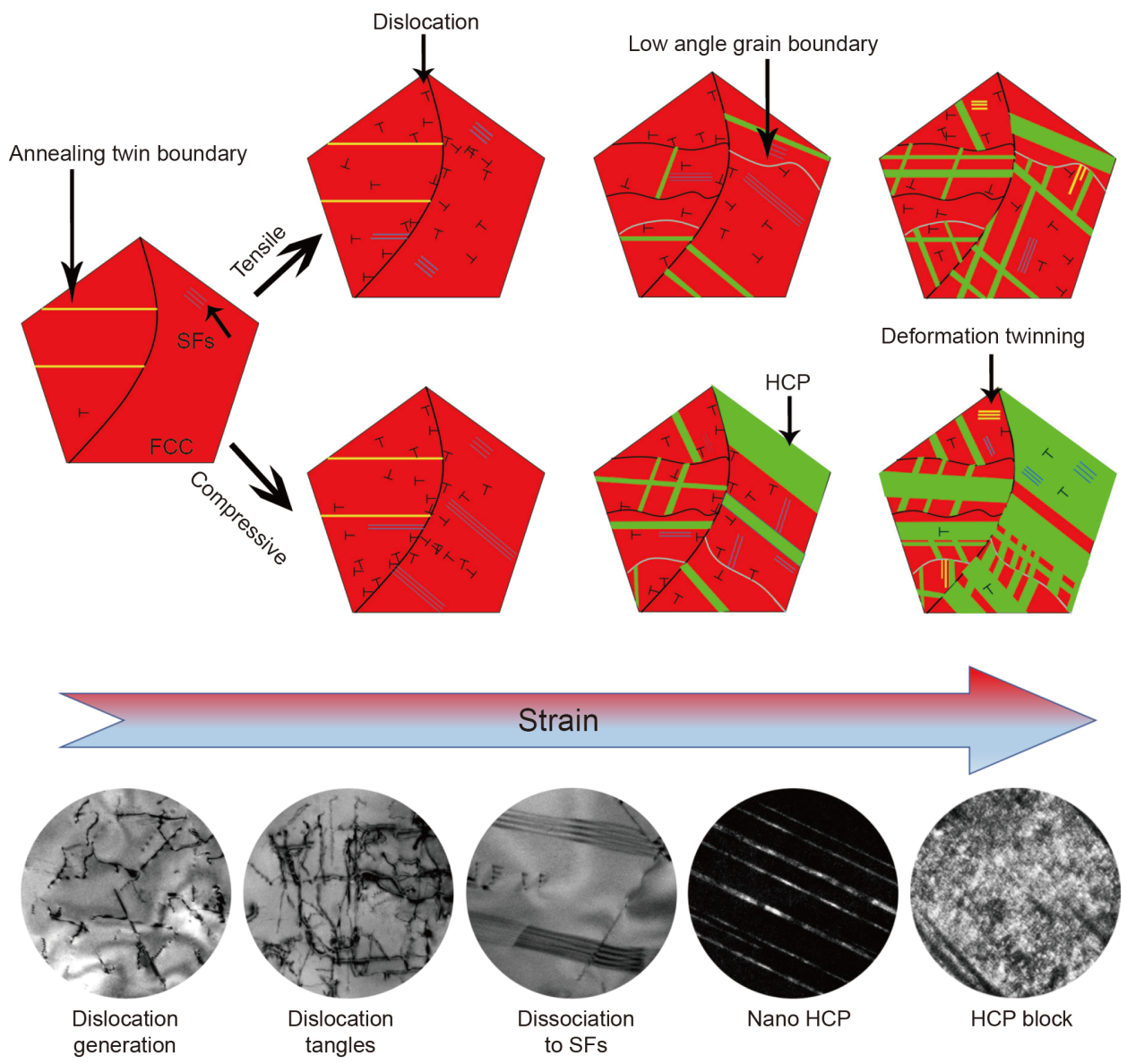

Figure 9 The schematic illustration and TEM images showing the deformation microstructure evolution of HEA under tension and compression. 
spectively. The resulting more intensive martensite phase transformation in compressive test significantly contributes to the strain hardening, the so-called TRIP effect [16], which leads to higher strain hardening rate. In the following, we will discuss the origin of this tensioncompression asymmetry in current HEA.

SFE is one of the most important intrinsic factors for martensite nucleation, since it determines the energy barrier [36]. The SFE of this HEA is $20 \mathrm{~mJ} \mathrm{~m}^{-2}$ which is close to the upper boundary of the TRIP region. The energy barrier between full dislocations and partial dislocations depends on the SFE. In our study, the planar slip dominates at the very early deformation stage, while the partial dislocations do not prevail, suggesting a high barrier between full dislocations and partial dislocations. Thus, more energy is required for the dissociation of dislocations in our HEA, causing the formation of martensite at medium and later stages of deformation. In previously reported metastable HEAs, such as $\mathrm{Fe}_{20} \mathrm{Co}_{30^{-}}$ $\mathrm{Cr}_{20} \mathrm{Ni}_{10} \mathrm{Mn}_{20}$ [8] and $\mathrm{Fe}_{49.5} \mathrm{Mn}_{30} \mathrm{Co}_{10} \mathrm{Cr}_{10} \mathrm{C}_{0.5}$ [37], the formation of $\mathrm{SFs}$ or martensite phase transformation dominates at the early deformation stage, due to their lower energy barriers between full dislocations and partial dislocations, stemming from lower SFEs (16 and $18 \mathrm{~mJ} \mathrm{~m}^{-2}$ ). As a result, higher extent of martensitic phase transformation is seen in these HEAs than present HEA. At the same tensile strain level (0.6), the fraction of martensite phase can reach over $35 \%$ in $\mathrm{Fe}_{49.5} \mathrm{Mn}_{30} \mathrm{Co}_{10^{-}}$ $\mathrm{Cr}_{10} \mathrm{C}_{0.5}$ [37], while this value is only $9 \%$ in our case.

Strain and stress are two critical extrinsic factors for the martensite phase transformation $[9,18]$. The strain provides the nucleation sites and the stress offers the driving force [38], both of which are essential for martensite phase transformation. In current study, at the same strain, the fraction of martensite is higher under compression than tension (see Fig. 4), suggesting that the tension-compression asymmetry results from the different applied stresses. Much higher stress in compression (see Table 1) provides stronger driving forces to overcome the martensite nucleation barrier, thus accounting for the formation of much more HCP phases. Especially for current HEA with a relative high SFE compared with other metastable HEAs, more driving force is required to trigger the martensite phase transformation. For instance, the stress of the compressive samples at the later deformation stage (strain of 0.6 ) is $\sim 500 \mathrm{MPa}$ higher than that of the tensile samples, resulting in the increment of $30 \%$ HCP phase. Similar phenomenon has been observed in literatures. Liu et al. [18] found martensite phase transformation in the FeCoCrNi HEA at low temperature tension (e.g., $4.2 \mathrm{~K}$ ) due to the higher applied stress, while the martensite was absence at room temperature. Wang et al. [21] reported that more martensites were formed at liquid nitrogen temperature than at room temperature under the same strain in the tensile tested $\mathrm{Fe}_{49.5} \mathrm{Mn}_{30}$ $\mathrm{Co}_{10} \mathrm{Cr}_{10} \mathrm{C}_{0.5}$ HEA. An important reason is the very high flow stress under the cryogenic conditions. In addition, when comparing the $\mathrm{T} 3$ and $\mathrm{C} 2$ samples with very close stress level $(\sim 1250 \mathrm{MPa})$, the fractions of HCP phase are nearly the same. This suggests that the martensite phase transformation is more sensitive to the applied stress in this HEA at relatively high strain levels. It is reasonable since sufficient nucleation sites were provided for phase transformation at very high strains. The stress became the dominating factor for martensite transformation. In short, a higher extent of martensite phase transformation during compression than tension in this HEA originates from the higher applied stress, which provides the larger driving force.

\section{CONCLUSION}

The microstructural evolution of a metastable FCC $\mathrm{Fe}_{40} \mathrm{Co}_{20} \mathrm{Cr}_{20} \mathrm{Ni}_{10} \mathrm{Mn}_{10}$ HEA deformed via tension and compression at various strain levels is investigated in present work. The main conclusions are as follows.

A significant tension-compression asymmetry in martensite phase transformation is shown. A higher content of HCP phases was detected under compression than tension, which arises from much higher stress that provides stronger driving forces.

Dislocation slip dominates the whole tensile regime, while martensite phase transformation occurs at the medium and later deformation stages. While for compression, dislocations only dominate the early deformation stage, yet impressive martensite takes place at the later stage. Twinning is occasionally found under both conditions.

In addition to the formation of HCP phase by overlapping SFs inside the grains, the HCP lamellae also form via partial dislocations emitted from low-angle grain boundaries, which serve as essential pathways of martensite phase transformation.

Received 25 February 2020; accepted 26 March 2020;

published online 29 May 2020

1 George EP, Raabe D, Ritchie RO. High-entropy alloys. Nat Rev Mater, 2019, 4: 515-534

2 Yeh JW, Chen SK, Lin SJ, et al. Nanostructured high-entropy alloys with multiple principal elements: Novel alloy design concepts and outcomes. Adv Eng Mater, 2004, 6: 299-303 
3 Lu Y, Gao X, Jiang L, et al. Directly cast bulk eutectic and neareutectic high entropy alloys with balanced strength and ductility in a wide temperature range. Acta Mater, 2017, 124: 143-150

4 Zhang Y, Zuo TT, Tang Z, et al. Microstructures and properties of high-entropy alloys. Prog Mater Sci, 2014, 61: 1-93

5 Liang YJ, Wang L, Wen Y, et al. High-content ductile coherent nanoprecipitates achieve ultrastrong high-entropy alloys. Nat Commun, 2018, 9: 4063

6 Shi P, Ren W, Zheng T, et al. Enhanced strength-ductility synergy in ultrafine-grained eutectic high-entropy alloys by inheriting microstructural lamellae. Nat Commun, 2019, 10: 489

7 Yang T, Zhao YL, Tong Y, et al. Multicomponent intermetallic nanoparticles and superb mechanical behaviors of complex alloys. Science, 2018, 362: 933-937

8 Liu SF, Wu Y, Wang HT, et al. Transformation-reinforced highentropy alloys with superior mechanical properties via tailoring stacking fault energy. J Alloys Compd, 2019, 792: 444-455

9 Li Z, Körmann F, Grabowski B, et al. Ab initio assisted design of quinary dual-phase high-entropy alloys with transformation-induced plasticity. Acta Mater, 2017, 136: 262-270

10 Wang Z, Baker I, Cai Z, et al. The effect of interstitial carbon on the mechanical properties and dislocation substructure evolution in $\mathrm{Fe}_{40.4} \mathrm{Ni}_{11.3} \mathrm{Mn}_{34.8} \mathrm{Al}_{7.5} \mathrm{Cr}_{6}$ high entropy alloys. Acta Mater, 2016, 120: $228-239$

11 Wang Z, Baker I, Guo W, et al. The effect of carbon on the microstructures, mechanical properties, and deformation mechanisms of thermo-mechanically treated $\mathrm{Fe}_{40.4} \mathrm{Ni}_{11.3} \mathrm{Mn}_{34.8} \mathrm{Al}_{7.5} \mathrm{Cr}_{6}$ high entropy alloys. Acta Mater, 2017, 126: 346-360

12 Song $\mathrm{M}$, Zhou $\mathrm{R}, \mathrm{Gu} J$, et al. Nitrogen induced heterogeneous structures overcome strength-ductility trade-off in an additively manufactured high-entropy alloy. Appl Mater Today, 2020, 18: 100498

13 Deng Y, Tasan CC, Pradeep KG, et al. Design of a twinninginduced plasticity high entropy alloy. Acta Mater, 2015, 94: 124133

14 Otto F, Dlouhý A, Somsen C, et al. The influences of temperature and microstructure on the tensile properties of a $\mathrm{CoCrFeMnNi}$ high-entropy alloy. Acta Mater, 2013, 61: 5743-5755

15 Wang Z, Guo L, Xia W, et al. An SEM-based approach to characterize the microstructural evolution in a gradient $\mathrm{CoCrFeNi}$ $\mathrm{Mo}_{0.15}$ high-entropy alloy. Mater Charact, 2020, 161: 110169

16 Li Z, Tasan CC, Pradeep KG, et al. A TRIP-assisted dual-phase high-entropy alloy: Grain size and phase fraction effects on deformation behavior. Acta Mater, 2017, 131: 323-335

17 Huang $\mathrm{H}, \mathrm{Wu} \mathrm{Y}, \mathrm{He}$ J, et al. Phase-transformation ductilization of brittle high-entropy alloys via metastability engineering. Adv Mater, 2017, 29: 1701678

18 Liu J, Guo X, Lin Q, et al. Excellent ductility and serration feature of metastable CoCrFeNi high-entropy alloy at extremely low temperatures. Sci China Mater, 2018, 62: 853-863

19 Li Z, Pradeep KG, Deng Y, et al. Metastable high-entropy dualphase alloys overcome the strength-ductility trade-off. Nature, 2016, 534: 227-230

20 Lu W, Liebscher CH, Dehm G, et al. Bidirectional transformation enables hierarchical nanolaminate dual-phase high-entropy alloys. Adv Mater, 2018, 30: 1804727

21 Wang Z, Lu W, Raabe D, et al. On the mechanism of extraordinary strain hardening in an interstitial high-entropy alloy under cryogenic conditions. J Alloys Compd, 2019, 781: 734-743

22 Chen S, Oh HS, Gludovatz B, et al. Real-time observations of
TRIP-induced ultrahigh strain hardening in a dual-phase CrMnFeCoNi high-entropy alloy. Nat Commun, 2020, 11: 826

$23 \mathrm{Su} \mathrm{J}, \mathrm{Wu} \mathrm{X}$, Raabe D, et al. Deformation-driven bidirectional transformation promotes bulk nanostructure formation in a metastable interstitial high entropy alloy. Acta Mater, 2019, 167: 2339

24 Liu SF, Wu Y, Wang HT, et al. Stacking fault energy of facecentered-cubic high entropy alloys. Intermetallics, 2018, 93: 269273

25 Gall K. The role of texture in tension-compression asymmetry in polycrystalline NiTi. Int J Plast, 1999, 15: 69-92

26 Liu Y, Xie Z, Van Humbeeck J, et al. Asymmetry of stress-strain curves under tension and compression for NiTi shape memory alloys. Acta Mater, 1998, 46: 4325-4338

27 Wu X, Tao N, Hong Y, et al. $\gamma \rightarrow \varepsilon$ martensite transformation and twinning deformation in fcc cobalt during surface mechanical attrition treatment. Scripta Mater, 2005, 52: 547-551

28 Wang B, An X, Huang Z, et al. Nitrogen doped Co-Cr-Mo-W based alloys fabricated by selective laser melting with enhanced strength and good ductility. J Alloys Compd, 2019, 785: 305-311

29 Lei $\mathrm{Z}, \mathrm{Liu} \mathrm{X}, \mathrm{Wu} \mathrm{Y}$, et al. Enhanced strength and ductility in a high-entropy alloy via ordered oxygen complexes. Nature, 2018, 563: 546-550

30 Yao MJ, Pradeep KG, Tasan CC, et al. A novel, single phase, nonequiatomic FeMnNiCoCr high-entropy alloy with exceptional phase stability and tensile ductility. Scripta Mater, 2014, 72-73: 5-8

31 Gutierrez-Urrutia I, Raabe D. Multistage strain hardening through dislocation substructure and twinning in a high strength and ductile weight-reduced Fe-Mn-Al-C steel. Acta Mater, 2012, 60: 5791-5802

32 Christian JW, Mahajan S. Deformation twinning. Prog Mater Sci, 1995, 39: 1-157

33 Zhu YT, Liao XZ, Wu XL. Deformation twinning in nanocrystalline materials. Prog Mater Sci, 2012, 57: 1-62

34 Chen M, Ma E, Hemker KJ, et al. Deformation twinning in nanocrystalline aluminum. Science, 2003, 300: 1275-1277

35 Zhu YT, Liao XZ, Srinivasan SG, et al. Nucleation and growth of deformation twins in nanocrystalline aluminum. Appl Phys Lett, 2004, 85: 5049-5051

36 Siegel DJ. Generalized stacking fault energies, ductilities, and twinnabilities of Ni and selected Ni alloys. Appl Phys Lett, 2005, 87: 121901

37 Li Z, Tasan CC, Springer H, et al. Interstitial atoms enable joint twinning and transformation induced plasticity in strong and ductile high-entropy alloys. Sci Rep, 2017, 7: 40704

38 Malard B, Pirling T, Inal K, et al. Stress determination during the mechanically-induced martensite phase transformation in the superelastic alloy CuAlBe by neutron diffraction. Mater Sci Forum, 2006, 524-525: 905-910

Acknowledgements This work was supported by the National Natural Science Foundation of China (51971247), and the open Foundation of State Key Laboratory of Powder Metallurgy at Central South University, Changsha, China.

Author contributions Wang $\mathrm{Z}$ and Song $\mathrm{M}$ designed the project. An $\mathrm{X}$ prepared the materials, conducted the mechanical testing, and performed the TEM. Wang Z performed the EBSD and ECCI. An X, Wang $\mathrm{Z}, \mathrm{Ni} \mathrm{S}$, and Song $\mathrm{M}$ analyzed the microstructural evolution. An X, Wang $\mathrm{Z}$, and Song $\mathrm{M}$ wrote the manuscript. All authors contributed to 
the discussion and commented on the manuscript.

Conflict of interest The authors declare that they have no conflict of interest.

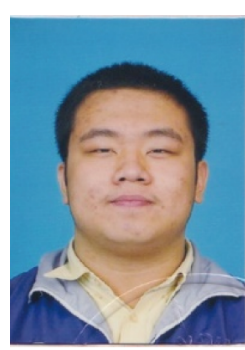

Xinglong An is currently a $\mathrm{PhD}$ candidate with Prof. Song Ni and Prof. Min Song at Powder Metallurgy Research Institute, Central South University, China. His current research focuses on the microstructure characterization of metallic materials.

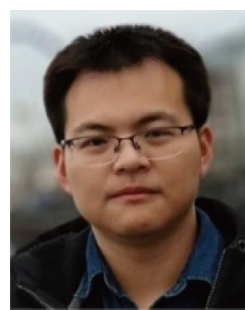

Zhangwei Wang is an Alexander von Humboldt fellow at the Max-Planck-Institut für Eisenforschung in Düsseldorf, Germany. He received his $\mathrm{PhD}$ degree in engineering from Dartmouth College, USA in 2017. His research primarily focuses on the development of advanced high-entropy alloys and lightweight steels.

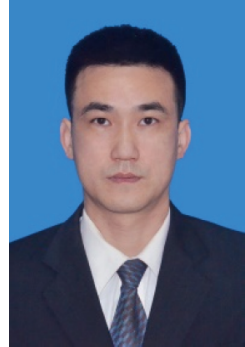

Min Song is a professor and Vise Dean of Powder Metallurgy Research Institute at Central South University. He serves as Associate Editor of "Materials Characterization". He received his $\mathrm{PhD}$ degree in 2005 at Dartmouth College, USA. His current research interests involve the deformation mechanisms of metallic materials, including: metals and alloys, bulk nanocrystalline materials, HEAs and metal matrix composites.

\section{亚稳 $\mathrm{Fe}_{40} \mathrm{Co}_{20} \mathrm{Cr}_{20} \mathrm{Mn}_{10} \mathrm{Ni}_{10}$ 高熵合金中马氏体的拉 伸压缩不对称性}

安星龙 ${ }^{1}$ 王章维 ${ }^{*}$, 倪颂 ${ }^{1}$ 宋旼 ${ }^{{ }^{*}}$

摘要 本文系统研究了具有面心立方结构的亚稳态 $\mathrm{Fe}_{40} \mathrm{Co}_{20} \mathrm{Cr}_{20}$ $\mathrm{Mn}_{10} \mathrm{Ni}_{10}$ 高熵合金在拉伸和压缩变形下的微观结构演变. 结果表 明, 从FCC到 HCP的马氏体相变在压缩条件下比在拉伸条件下要 高得多, 具有明显的拉伸-压缩不对称性. 与拉伸试验相比, 压缩试 验的真应力更大, 为马氏体相变的开启提供了更大的驱动力. 除马 氏体相变外，位错的平面滑移以及少量的变形孪晶在拉伸和压缩 过程中均有发现. 位错滑移在整个拉伸变形过程中起主导作用, 而 在压缩变形中位错运动和马氏体相变都起到了关键作用. 由于该 高熵合金具有中等的层错能 $\left(20 \mathrm{~mJ} \mathrm{~m}^{-2}\right)$, 因此马氏体相变更倾向 于在晶界或亚晶界等高能区域处成核. 我们发现了六方马氏体相 可以通过从小角晶界发射不全位错来形成，这为马氏体相变提供 了新的路径. 本研究对于理解亚稳态高熵合金的变形机制具有重 要的意义. 